\title{
Spatial heterogeneities in tectonic stress in Kyushu, Japan and their relation to a major shear zone
}

\author{
Satoshi Matsumoto ${ }^{1 *}$, Shigeru Nakao ${ }^{2}$, Takahiro Ohkura ${ }^{3}$, Masahiro Miyazaki ${ }^{1}$, Hiroshi Shimizu ${ }^{1}$, Yuki Abe ${ }^{4}$, \\ Hiroyuki Inoue ${ }^{3}$, Manami Nakamoto', Shin Yoshikawa ${ }^{3}$ and Yusuke Yamashita ${ }^{4}$
}

\begin{abstract}
We investigated the spatial variation in the stress fields of Kyushu Island, southwestern Japan. Kyushu Island is characterized by active volcanoes (Aso, Unzen, Kirishima, and Sakurajima) and a shear zone (western extension of the median tectonic line). Shallow earthquakes frequently occur not only along active faults but also in the central region of the island, which is characterized by active volcanoes. We evaluated the focal mechanisms of the shallow earthquakes on Kyushu Island to determine the relative deviatoric stress field. Generally, the stress field was estimated by using the method proposed by Hardebeck and Michael (2006) for the strike-slip regime in this area. The minimum principal compression stress $(\sigma 3)$, with its near north-south trend, is dominant throughout the entire region. However, the $\sigma_{3}$ axes around the shear zone are rotated normal to the zone. This result is indicative of shear stress reduction at the zone and is consistent with the right-lateral fault behavior along the zone detected by a strain-rate field analysis with global positioning system data. Conversely, the stress field of the normal fault is dominant in the Beppu-Shimabara area, which is located in the central part of the island. This result and the direction of 03 are consistent with the formation of a graben structure in the area.
\end{abstract}

Keywords: Stress field; Kyushu; Shear zone; Graben

\section{Findings}

Introduction

A stress field is an important parameter for understanding the dynamics of the Earth's crust and earthquake occurrence. Following the development of techniques that can estimate the stress field from the focal mechanism data of earthquakes (Angelier 1979, 1984; Michael 1984), many studies have estimated the relative deviatoric stress fields at various tectonic settings. These studies have concluded that the stress field reveals heterogeneous features that relate to the loading processes acting on the medium. This spatial variation of the stress field is attributed to both non-uniform mechanical forces acting on the outer boundary of the medium and/or the inelastic behavior of the medium interior, such as during faulting and magma intrusion (Matsumoto et al. 2012), both

\footnotetext{
* Correspondence: matumoto@sevo.kyushu-u.ac.jp

${ }^{1}$ Institute of Seismology and Volcanology, Faculty of Sciences, Kyushu

University, Shimabara, Japan

Full list of author information is available at the end of the article
}

of which are possible causes of spatial variation in the observed seismicity. For instance, the spatial and temporal changes in a stress field can be attributed to the behavior of a shear zone such as the San Andreas Fault zone (Zoback et al. 1987; Hardebeck and Hauksson 2001; Townend and Zoback 2001, 2004; Hardebeck and Michael 2004). Therefore, detailed estimation of the stress field can facilitate the understanding of tectonic processes.

Kyushu Island is located in the southwestern part of Japan. It lies on the overriding plate above the subducting Philippine Sea slab (PHS) to the west of the Nankai Trough subduction zone. A large right-lateral shear zone running through the central part of Kyushu Island divides the island in terms of its geologic features. The shear zone is an extension of the median tectonic line (MTL) that originates at Honshu Island and transects Shikoku. The MTL is the largest tectonic line in southwestern Japan and is mainly composed of metamorphic rocks. In the present-day tectonic setting, geological

\section{实 Springer}


studies have reported right-lateral strike-slip and extensional movements along the MTL on Kyushu Island (e.g., Okada 1980; Kamata and Kodama 1994, 1999). An area transecting in the EW direction at the central part of Kyushu, which is located to the north of the MTL from the city of Beppu to the Unzen Volcano at Shimabara Peninsula, is called the Beppu-Shimabara graben (hereafter, $\mathrm{B}-\mathrm{U}$ area) owing to the many normal faults around and between Beppu and Unzen. Itoh et al. (2014) investigated the basin-forming process at the Beppu region, which revealed that the major structure was halfgraben under extensional stress in $\mathrm{N}-\mathrm{S}$, and PHS convergence strongly affected formation in the region.

The strain-rate field on Kyushu has been modeled by block inversion of global positioning system (GPS) data (Nishimura and Hashimoto 2006; Wallace et al. 2009). These studies found that the major factor controlling the strain-rate field was the subduction of the PHS. In addition, relative movement between the blocks has been detected. The shear zone at the southern edge of the $\mathrm{B}-\mathrm{U}$ area is one of the block interfaces in Kyushu Island characterized by right-lateral motion, and it moves approximately $7 \mathrm{~mm} / \mathrm{y}$ (Wallace et al. 2009). In this study, we have redefined the location of the shear zone at the southern end of the active fault in the $\mathrm{B}-\mathrm{U}$ area as shown in Fig. 1. This zone consists of both the Oita-Kumamoto and Usuki-Yatsushiro tectonic lines (e.g., Kimura et al. 1993). Both these lines are boundaries of major geologic terranes that constitute the basement of Kyushu Island, and they behave as rightlateral strike-slip faults (National Institute of Advanced Industrial Science and Technology 2013).
Kyushu Island is one of the regions in Japan that hosts significant volcanic activity. The volcanic front runs through the central part of the island from north to south, and a group of volcanoes is also located on a line obliquely crossing the volcanic front. The active volcanoes in this group are Beppu, Kuju, Aso, and Unzen. Their locations are shown in Fig. 1. This figure also shows active faults mapped by the National Institute of Advanced Industrial Science and Technology (hereafter, AIST) as well as the epicenter distribution of microearthquakes with depths of less than $30 \mathrm{~km}$. As seen in Fig. 1, the main areas with high seismicity of microearthquakes are the B-U area and the northern offshore area of the island. The high activity observed in the northern part corresponds to the aftershock area of the M7.0 2005 Fukuoka earthquake (Shimizu et al. 2006). The seismic activity in the central part of Kyushu Island remains stable and high, thus requiring several intrinsic conditions of stress and/or strength of the medium to explain the occurrence of earthquakes. Townend and Zoback (2006) and Terakawa and Matsu'ura (2010) found that the dominant direction of minimum principal stress $\left(\sigma_{3}\right)$ generally trends $\mathrm{N}-\mathrm{S}$ in southwestern Japan. However, the small amount of data from Kyushu Island has only provided a spatial resolution of several tens of kilometers. Therefore, we have attempted to estimate the local stress field at a higher resolution with significant quantities of data on the focal mechanisms in this area.

\section{Focal mechanisms and the stress field at Kyushu Island}

We used focal mechanisms to determine the stress condition in the seismogenic layer. Shallow earthquakes

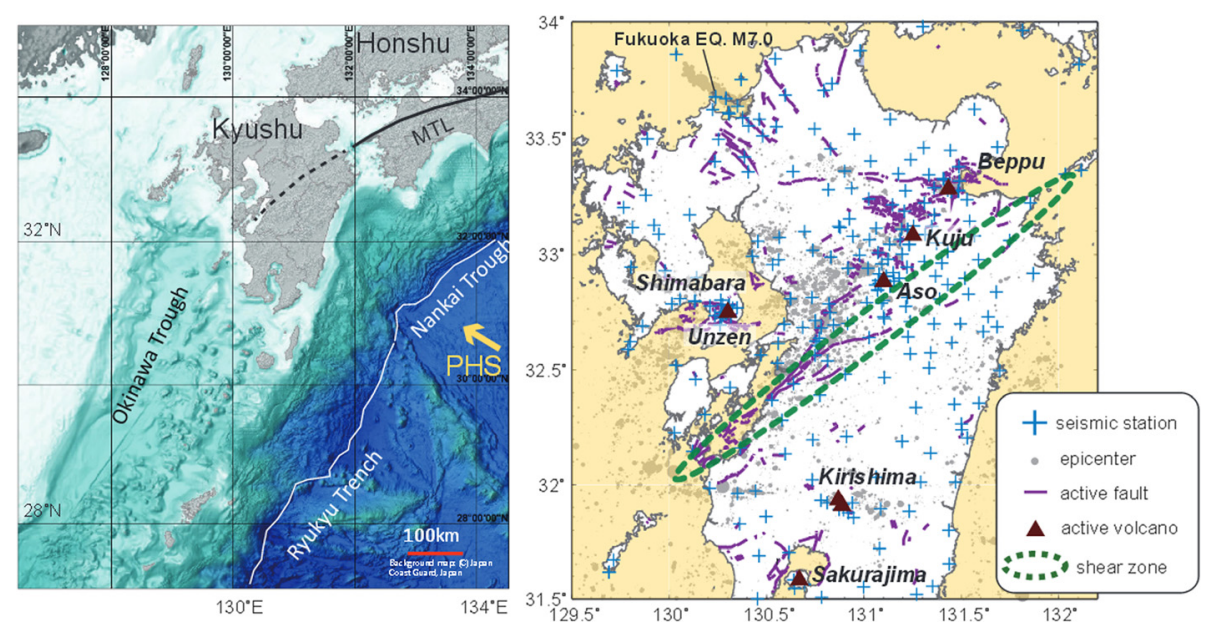

Fig. 1 Map showing (left) the location of Kyushu Island and (right) the seismic stations and hypocenters of earthquakes originating at depths shallower than $30 \mathrm{~km}$. Events indicated by gray circles occurred from January 1993 to August 2011. The size of the marker is proportional to its magnitude, as shown in the lower-left scale. Crosses denote seismic stations that recorded the data used in this analysis. Triangles and purple segments indicate the active volcanoes and active faults in the study area, respectively. The Philippine Sea slab (PHS) is subducting under the Nankai Trough or the Ryukyu Trench in the direction of the yellow arrow in the map on the left-hand side 
with magnitudes greater than 1.0 that occurred at depths ranging from 0 to $30 \mathrm{~km}$ from January 1993 to July 2013 were analyzed in this study. The locations of the seismic stations used in the analysis have been plotted in Fig. 1. The stations were installed by Kyushu, Kyoto, and Kagoshima Universities, by Hi-net of the National Research Institute for Earth Science and Disaster Prevention (NIED), and by the Japan Meteorological Agency (JMA); several stations were set up temporarily to determine the focal mechanisms with high accuracy. Most of the temporal seismic stations have been installed since 2005. The hypocenters in this area, with the exception of the Fukuoka earthquake aftershocks, can be determined by using three-dimensional velocity structures obtained through the tomographic inversion of travel-time data (Saiga et al. 2010). The hypocenter for the Fukuoka area was determined by the velocity structure of Hori et al. (2006) because those data provided a finer structure after high-aftershock activity in the area as compared to the data of Saiga et al. (2010). As many as 40,981 hypocenters have been located over approximately 20 years. A focal mechanism was estimated from the polarity data on the onset of the first $\mathrm{P}$ wave at eight or more stations by using the HASH algorithm of Hardebeck and Shearer (2002). Only the focal mechanisms characterized by a low misfit of the algorithm (i.e., $L<0.2$, where $L$ is the misfit ratio to the total number of polarity data with a weighting factor calculated by the $\mathrm{P}$ wave amplitude of the focal mechanism, and $\mathrm{S}<0.4$, where $S$ is the sum of the weighting factors per the total number of polarity data) were used in this study. A total of 9177 focal mechanisms were adopted for the stress analysis.

Figure 2 indicates the orientation of the compression (P) and tension (T) axes for earthquakes at depths shallower than $30 \mathrm{~km}$. As can be seen in Fig. 2, the major fault type on Kyushu Island is strike-slip. In particular, most of the seismic motions at the hypocentral area of the Fukuoka earthquake and around the shear zone at the southern edge of the $\mathrm{B}-\mathrm{U}$ area were strike-slip. In contrast, many events of the normal-fault type were found in the B-U area. The change in the style of faulting from strike-slip to normal, as shown by the focal mechanisms, can be attributed to either the strengthening of the vertical stress or the weakening of the maximum horizontal stress in the medium. Figure 2 shows that the P- and T-axes are generally trending WSWENE and NNW-SSE, respectively. In addition, these general directions distort and rotate clockwise around the $\mathrm{B}-\mathrm{U}$ area. This trend reinforces the possibility that the boundary conditions around Kyushu Island are laterally variable, and/or several internal structures in the crust can change the stress field in the medium.

In this study, we have analyzed the available data under the assumption that an individual focal mechanism is controlled by a slip on a pre-existing fault under the local tectonic stress field. We have estimated the two-dimensional stress field on Kyushu Island by using the stress-tensor inversion method of Hardebeck and Michael (2006). The stress-tensor inversion method permits an estimation of the principal directions of the
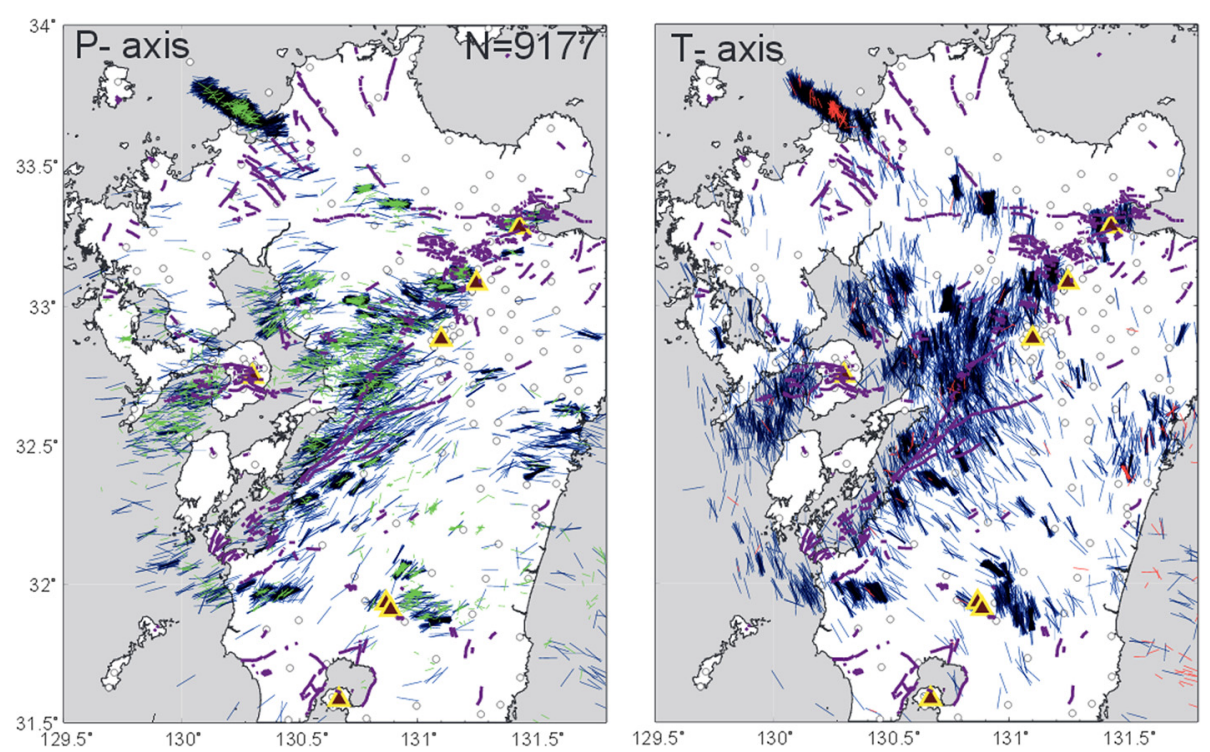

Fig. $2 \mathrm{P}$ - and T-axes adopted in the stress-tensor inversion. (Left) P-axis direction is indicated by the segments. Blue and green segments represent P-axes plunging $\leq 45^{\circ}$ and $>45^{\circ}$ from the horizontal (e.g., strike-slip and normal-fault type), respectively. (Right) Distribution of the T-axes of the focal mechanisms is shown by the segments. Red segments represent reverse faulting events. Open circles denote the locations of the seismic stations. Other symbols are the same as in Fig. 1 
tectonic stress tensor and stress ratio, and the relevant equation is as follows:

$$
\phi=\left(\sigma_{2}-\sigma_{3}\right) /\left(\sigma_{1}-\sigma_{3}\right),
$$

where $\sigma_{1}, \sigma_{2}$, and $\sigma_{3}$ are the maximum, moderate, and minimum compressional principal stresses, respectively. A relative deviatoric stress has been estimated from the data of the seismic slip direction that coincides with that of the shear traction acting on the pre-existing fault since its absolute value is not able to be obtained from only slip direction data. Hardebeck and Michael (2006) developed a method to estimate the spatial variation of the stress field from parametric data gathered at spatially distributed grid points. In this study, we have adopted their algorithm to reveal the heterogeneous structure of the stress field on Kyushu Island. The relative deviatoric stress tensors were estimated at grid points set at latitudinal and longitudinal intervals of $0.15^{\circ}$. We have assumed a heterogeneous stress field on a scale larger than approximately $15 \mathrm{~km}$. The resolution in this study was, therefore, chosen to be $15 \mathrm{~km}$. Stress in the crust is known to increase with depth because of overburden pressure. Therefore, the relative deviatoric stress field also could vary in the vertical direction. However, the depth distribution of earthquakes is not always uniform; instead, it is typically clustered. Thus, the resolution of the stress inversion reduces with depth, and therefore, a two-dimensional relative deviatoric stress field has been assumed in this analysis. This assumption is applicable for the stabilization of parameter estimations in the inversion.

The principal directions of the stress tensor at each grid point have been obtained and are plotted in Fig. 3a. Specifically, the two directions closest to the horizontal plane have been plotted. Here, we define maximum and minimum principal stress in the two directions as $\sigma_{\mathrm{Hmax}}$ and $\sigma_{\mathrm{Hmin}}$, respectively. At most of the grid points, the moderate principal stress axis is in the vertical direction. The black segments in Fig. 3a show the results at the grid points where the directions of $\sigma_{1}$ and $\sigma_{2}$ could not be distinguished. The stress tensor estimations for $\sigma_{3}$ at most of the grid points were well resolved, and they are oriented horizontally. Directions of the principal axes generally reveal the regional features; the direction of $\sigma_{3}$ in the $\mathrm{B}-\mathrm{U}$ area is $\mathrm{N}-\mathrm{S}$, and it is oriented to NNW-SSE or NW-SE in other regions. The $\sigma_{3}$ directions seem to be normal to the strike of the active fault in the shear zone at the southern boundary of the B-U area. Figure $3 \mathrm{~b}$ shows the stress ratio $\phi$ ' at each of the distributed grid points, with the radius of the circle proportioned to the estimated stress ratio. In this study, we have converted the stress ratio $\phi$ to $\phi$, which is defined as $\phi^{\prime}=\left(\sigma_{\mathrm{H} \max }-\sigma_{\mathrm{Hmin}}\right) /\left(\sigma_{\mathrm{v}}-\sigma_{\mathrm{H} \min }\right)$, where $\sigma_{\mathrm{H} \max }, \sigma_{\mathrm{Hmin}}$, and $\sigma_{\mathrm{v}}$ are the maximum and minimum horizontal and vertical principal stresses, respectively. This conversion was performed to distinguish the strike-slip stress regime from the normal stress one. The range between the
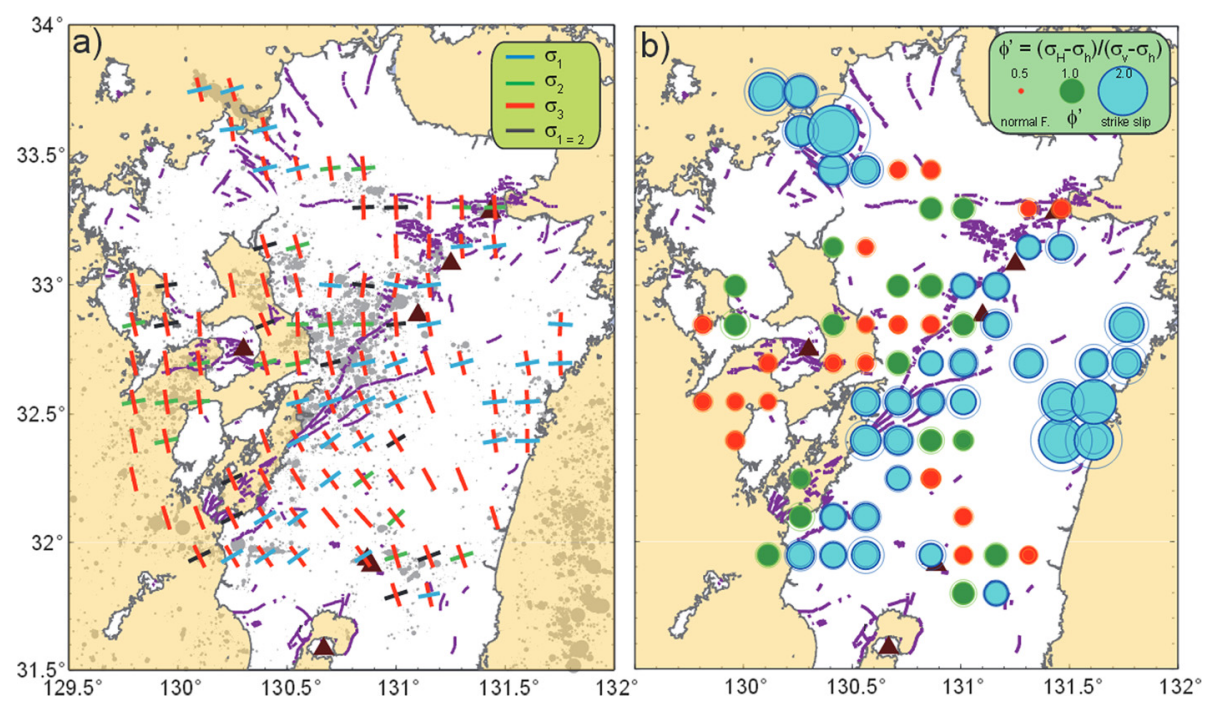

Fig. 3 a Principal direction of the stress tensor at the grid points. Two of the eigen directions of the stress tensor close to the horizontal plane are plotted. The blue and red segments are the directions of $\sigma_{1}$ and $\sigma_{3}$, respectively, and the green segment shows the moderate principal stress direction of $\sigma_{2}$. The black segment indicates the stress condition with $\sigma_{2} \approx \sigma_{1}$ from the obtained stress ratio. Gray circles denote the hypocenters in this area. $\mathbf{b}$ Stress ratios $\phi^{\prime}=\left(\sigma_{H \max }-\sigma_{H \min }\right) /\left(\sigma_{v}-\sigma_{H \min }\right)$ at the grid points. Blue, green, and red circles denote stress ratio ranges $\phi^{\prime}>1.05$, $1.05-0.95$, and $<0.95$, respectively; their radii are proportional to the ratio. The outer and inner circles of the estimated value indicate the $95 \%$ confidence range 
outer and inner circles indicates that the values within the $95 \%$ confidence range have been estimated by the bootstrap test described below.

A bootstrap test that iteratively resamples the focal mechanism dataset and checks the solution distribution has been performed to examine the reliability of the result. Figure 4 shows the results of the test at each grid point. The $\sigma_{3}$ directions were resolved well, as can be seen in Fig. 4. However, the range of $\sigma_{1}$ overlapped with $\sigma_{2}$ at some grid points. Especially, this can be seen at the grid in the B-U area. This implies that the solutions of $\sigma_{1}$ at those grid points could not be separately estimated from $\sigma_{2}$. This kind of result is typical for a high stress ratio $\left(\sigma_{1} \approx \sigma_{2}\right)$. The small stress ratio $\phi^{\prime}$ found in the western $\mathrm{B}-\mathrm{U}$ area implies that the $\sigma_{\mathrm{H} \max }$ values are very close to or smaller than $\sigma_{\mathrm{v}}$. This outcome can also be seen in the mechanism distribution pattern in areas where normal faulting occurs frequently. A small stress ratio implies that $\sigma_{\mathrm{Hmax}}$ is weakened in the area around the grid point because the vertical stress $\left(\sigma_{\mathrm{v}}\right)$ is basically dependent on the overburden pressure and would, therefore, be uniform compared with the horizontal stress. In contrast, large ratios of approximately 0.6 were found in the areas near the Fukuoka earthquake faults.

\section{Discussion}

\section{Stress regimes at Kyushu}

As seen in Figs. 3a and 4, the minimum principal compression is stable over the entire island of Kyushu. The dominant $\sigma_{3}$ direction implies that Kyushu Island is undergoing extensional tectonics at present. According to a geological study by Kamata and Kodama (1999), this extensional feature can be attributed to the behavior of the Ryukyu Trench, which is located southeast of Kyushu Island, and this trench might act relatively as an extension force on Kyushu by its roll-back. The behavior of the trench possibly induces seafloor spreading at the Okinawa Trough, northeast of the trench. The direction of the spreading is NNW-SSE, which could also be the reason behind the dominance of $\sigma_{\mathrm{Hmin}}$ at Kyushu Island.

On the basis of the stress field characteristics, the island can be divided into the following three regions: the north area, the $\mathrm{B}-\mathrm{U}$ area, and the south area. The northern region, which is defined as the area north of $33.3^{\circ} \mathrm{N}$, is dominantly a strike-slip regime, and $\sigma_{\mathrm{Hmin}}$ is in the NNW-SSE direction. Most of the active fault traces (purple segments in Fig. 3) are oriented toward a favorable direction for shear faulting. Shear stress is especially large in the Fukuoka earthquake area. The northern B$\mathrm{U}$ area might be bounded by the faults near $33.3^{\circ} \mathrm{N}$ that strike E-W, and the southern part is bounded by the MTL shear zone. The stress field in the B-U area is represented by $\sigma_{\mathrm{Hmin}}$ in the $\mathrm{N}-\mathrm{S}$ direction and is dominantly characterized by normal faults. This trend is consistent with the direction of the formation of the graben structure seen in the B-U area. Although the normal faults at the surface only appear in the Hohi volcanic zone (around the Beppu region) and the Unzen area (Kamata and Kodama 1994), the entire B-U area is under the stress regime characteristic of normal faults.

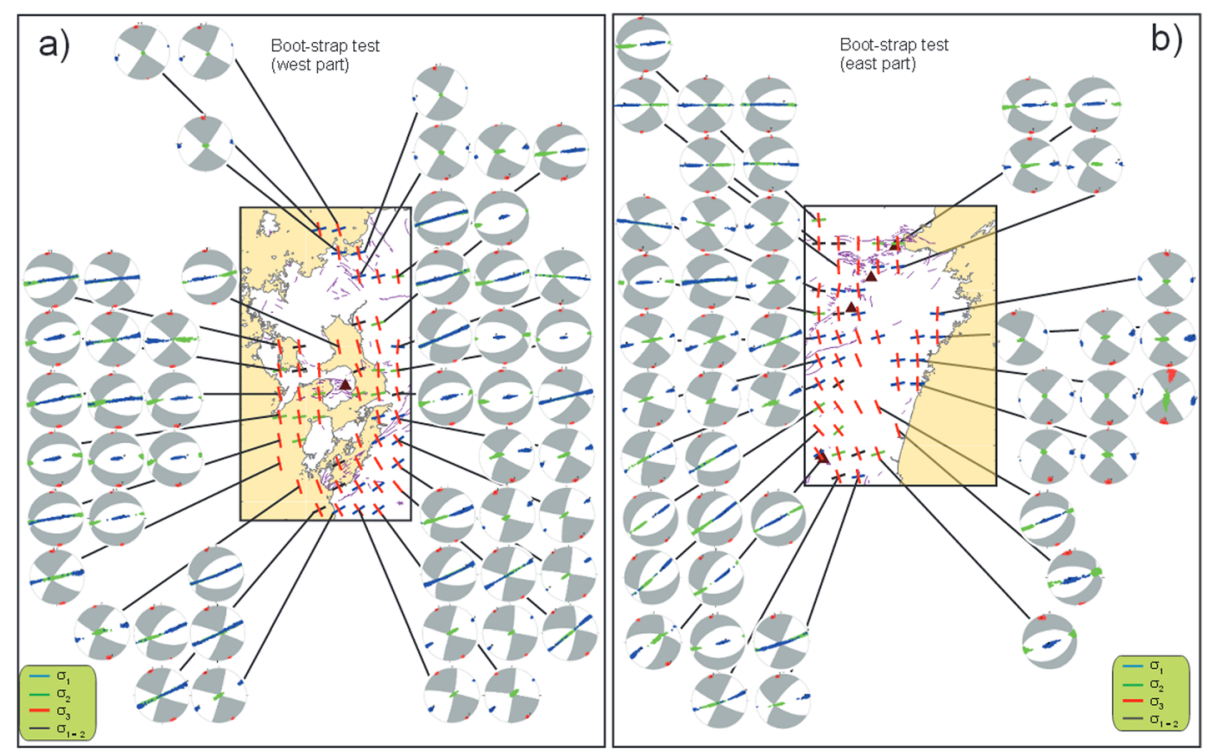

Fig. 4 Principal stress directions at the spatial grid within the $95 \%$ confidence range by the bootstrap test. $\mathbf{a}$ and $\mathbf{b}$ are the western and eastern parts of Kyushu. The direction is plotted on the lower hemisphere. The optimum stress field at each grid is shown as a focal mechanism plot. Blue, green, and red dots indicate $\sigma_{1}, \sigma_{2}$, and $\sigma_{3}$, respectively. Each "beach ball" location corresponds to a spatial grid-point distribution on the map at the center of the figure. The map shows a part of Fig. $3 \mathrm{a}$ 
In addition, the $\sigma_{\mathrm{Hmin}}$ direction varies from $\mathrm{N}-\mathrm{S}$ to NNW-SSE toward the shear zone. The southern area lies to the south of the shear zone. In this area, the direction of $\sigma_{\mathrm{Hmin}}$ is either NNW-SSE or NW-SE. The $\sigma_{\mathrm{Hmin}}$ directions at the shear zone tend to be normal to the strike of the active faults.

According to a paleostress study in the southern area (Yamaji 2003), $\sigma_{\text {Hmin }}$ was acting in the WNW-ESE direction during Late Pliocene. The present-day stress has an opposite sense, thus indicating that the extensional tectonics of the Late Pliocene have terminated and changed to compression along the WNW-ESE direction. Yamaji (2003) suggested that a tectonic event, such as a change in the direction of subduction of the PHS from NNW to NW, led to this change. This change then enhanced the collision between Sikoku and Kyushu because the subduction rate of the PHS increased. Seno (1999) investigated the stress field in Kyushu and pointed out that the spatial characteristics of the stress field could not be explained by simple plate interactions. Basically, the stress profile he proposed is similar to the stress field suggested in this study. However, there are some differences from his model, which involve the stress regime of the normal stress in the Beppu area and the strike-slip movement in western Kyushu. According to our results, the normal stress regime in Beppu is still functional. Minimum principal stress is oriented along the N-S or NNW-SSE direction over all of Kyushu Island.

Figure 5 shows the principal stress directions plotted on a map of the principal strain rate, which was estimated by using 5 years of GPS observation data collected by Nakao et al. (2005). Strain fields around volcanoes such as Kirishima and Sakurajima are strongly perturbed because of volcanic activity. Here, we have excluded these areas from the discussion because of the short-term variation in the effects of volcanic activity on the strain field. In Fig. 5, we can see the difference between the shear strain rate and the stress directions. This finding shows that the short-term deformation detected by the GPS survey does not always represent stress conditions in Kyushu. According to Wallace et al. (2009), the subduction of the PHS is responsible for the strain-rate field. The subduction directly affects the strain field in the eastern part of the southern area. The strain rate is on the order of $10^{-7}$ per year, which means that the stress increment is approximately $3 \mathrm{kPa}$ when the rigidity is $30 \mathrm{GPa}$. This value is much smaller than the stress drop of an earthquake (i.e., approximately $20 \mathrm{MPa}$ ). Increasing the stress level to nucleate an earthquake might take several thousand years. However, great earthquakes have occurred along the plate interface at the Nankai Trough, in southwest Japan, at intervals of approximately 100 years. This cycle suggests that the

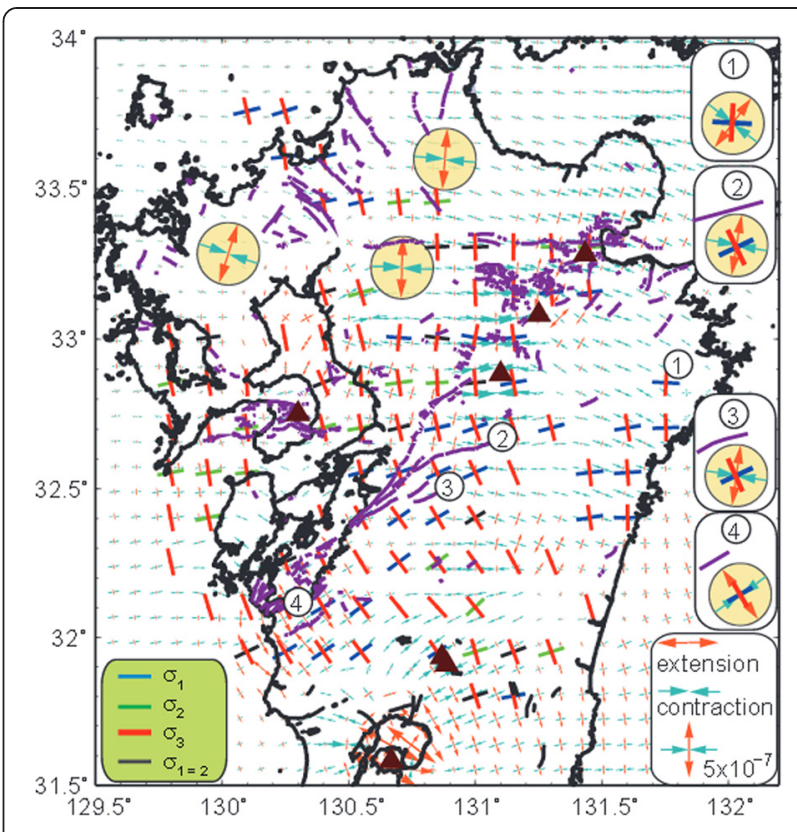

Fig. 5 Principal stress directions and the principal strain observed over 5 years by Nakao et al. (2005). The principal stress map is the same as in Fig. 4a. The vector of the principal strain is plotted as shown in the legend. Arrows in a yellow circle on the map denote the direction of typical principal strain at the location of the circle. Stress, strain, and active faults at the points with numerals on the map are plotted at the left of the map to show their directions in detail

stress loads caused by locking of the subduction at the interface could be released and associated with the earthquake occurrence every hundred years. Therefore, the coupling at the interface between the inland plate and the PHS is not sufficient to create stress to generate earthquake activity in Kyushu, as pointed out in central Japan by Townend and Zoback (2006).

\section{Decreasing shear stress at the MTL on Kyushu}

The MTL is a geologic boundary, which has been undergoing right-lateral motion since the Quaternary Period (e.g., Kamata and Kodama 1994). At this geologic boundary, the seismic/aseismic slip could transition to a "shear zone." We have found that the direction of $\sigma_{\mathrm{Hmin}}$ is normal to the shear zone at the boundary between the $\mathrm{B}-\mathrm{U}$ and the southern area, which indicates that the shear stress does not act on the faults in the MTL. The $\sigma_{\text {Hmin }}$ gradually changes its direction to normal to the shear zone from both the B-U and the southern area. A possible interpretation for this change is that the shear zone releases shear stress as a weak zone. In addition, there is a difference between the shear strain rate and the stress directions in the southern area, as indicated by the extracted points 1, 2, and 3 in Fig. 5. The shear strains in the extracted points are obliquely oriented to the fault, which could produce shear stresses on the 
shear zone at present. However, the principal stress directions exhibit no shear stress on the fault. This suggests that the shear zone behaves as a shear stress reduction system. Wallace et al. (2009) estimated that there is a dextral displacement of $7 \mathrm{~mm} / \mathrm{y}$ at the MTL in Kyushu by using GPS data at the shear zone. Two factors, namely (1) geodetically detected right-lateral motion at the shear zone and (2) perpendicular $\sigma_{\mathrm{Hmin}}$ to the shear zone, provide a reasonable explanation for the interpreted reduction in shear stress at this zone. The shear stress reduction at the zone results in an increase in $\sigma_{\mathrm{Hmin}}$ and decrease in $\sigma_{\mathrm{Hmax}}$, which could create a stress regime characteristic of normal faults. However, the stress regime at the shear zone is still the strike-slip type. This implies that $\sigma_{\mathrm{H} \max }$ is higher than $\sigma_{\mathrm{v}}$ even as differential stress is reduced by right-lateral slip at the shear zone. Therefore, we need to consider other mechanisms that may have caused the normal stress regime in the $\mathrm{B}-\mathrm{U}$ area (i.e., $\sigma_{\mathrm{v}}$ becomes the maximum principal stress).

\section{Stress field in the $B-U$ area}

As mentioned earlier, the estimated stress fields in the northern and southern areas are characterized by strikeslip regimes with $\sigma_{\mathrm{Hmin}}$ in the NW-SE or NNW-SSE direction. In contrast, $\sigma_{\mathrm{Hmin}}$ is oriented $\mathrm{N}-\mathrm{S}$ at the $\mathrm{B}-\mathrm{U}$ area. In addition, the stress ratio $\phi^{\prime}$ in the $\mathrm{B}-\mathrm{U}$ area reveals that $\sigma_{\text {Hmax }}$ is equal to or smaller than $\sigma_{v}$. The change in stress regime in the $\mathrm{B}-\mathrm{U}$ from both the northern and southern area requires a declining mechanism of $\sigma_{\mathrm{Hmax}}$ in this area. The shear stress reduction at the shear zone is insufficient to decrease $\sigma_{\mathrm{Hmax}}$ down to $\sigma_{\mathrm{v}}$ as described above.

In Fig. 5, the principal strain directions are similar to the stress directions in the $\mathrm{B}-\mathrm{U}$ area. In addition, an area with a large contraction in the $\mathrm{E}-\mathrm{W}$ direction can be found around the Aso and Kuju volcanoes. This large contraction might relate to volcanic activity of the volcanoes and indicates the possibility of the existence of a mechanical anomalous structure. For example, in the case that the area is filled up with material with a low elastic coefficient, the area could behave as a weak zone. This is a possible cause for the decline of $\sigma_{\mathrm{Hmax}}$. The principal strain-rate-oriented E-W corresponds to the $\sigma_{\text {Hmax }}$ direction. This relation suggests that linear elastic behavior dominates in the surrounding area of the large contraction, although the value of stress cannot directly compare to the strain rate. Therefore, strengths of $\sigma_{\mathrm{Hmax}}$ east and west of the area could be low because of the contraction.

Figure 6 shows a schematic illustration of the stress conditions on Kyushu and the Bouguer anomaly. Active

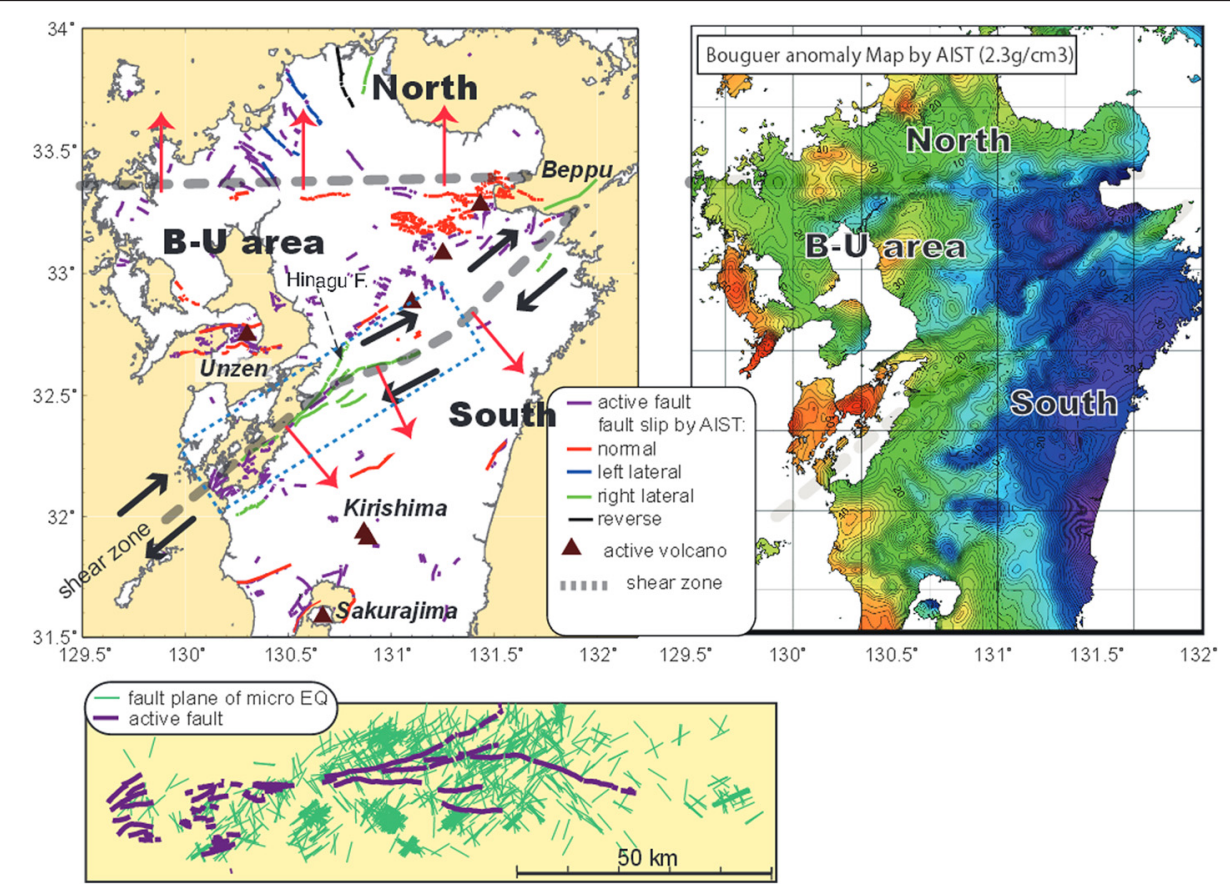

Fig. 6 (Upper left) Schematic illustration of the stress field and shear zone at Kyushu Island. Behavior of the active fault, as determined by the AIST, is plotted by the color segments that are defined in the legend. Solid arrows indicate the direction of the behavior of the shear zone. Red arrows show the extensional force acting on the B-U area. (Upper right) Bouguer anomaly map assuming a terrain density of $2.3 \mathrm{~g} / \mathrm{cm}^{3}$ (copyright for the basemaps of the gravity maps belongs to the Geospatial Information Authority of Japan (GSI); the map is licensed under the Creative Commons Attribution 2.1 Japan License). (Lower) Fault planes of micro-earthquakes (green) and active fault traces (purple) in the area surrounded by the blue dashed line in the upper figure. The planes were found to be oblique to the active fault traces 


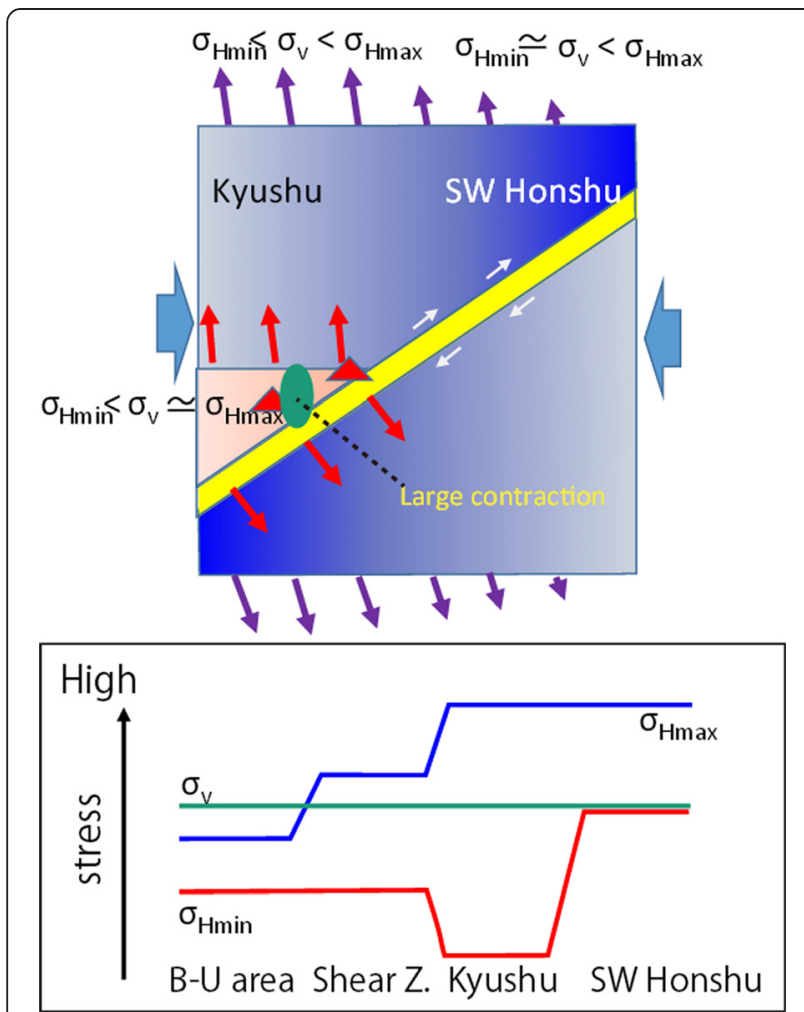

Fig. 7 Schematic illustration of a model of the stress field. (Upper) Stress regime and horizontal principal direction of the stress from southwest Honshu to Kyushu. Yellow and light orange areas indicate the shear zone and $\mathrm{B}-\mathrm{U}$ area, respectively. The slip direction at the shear zone is indicated by white arrows. The green ellipsoid in the $\mathrm{B}-\mathrm{U}$ area implies an area with large contraction. Red triangles indicate volcanoes in the Aso and Beppu regions. Red and purple arrows show directions for $\sigma_{H \min }$. The $\sigma_{H \max }$ direction is shown by the thick right blue arrow. (Lower) Spatial principal stress variation from the $\mathrm{B}-\mathrm{U}$ area to southwest Honshu

faults are categorized as normal, left- and right-lateral, and reverse by the AIST (2013); these have been indicated by red, blue, green, and black segments, respectively. The purple segment is also an active fault, although its behavior has not been clearly determined. Most of the shear zones are associated with right-lateral faults. The B-U area mostly corresponds to the lowgravity anomaly zone, which is indicative of a graben structure. A large number of micro-earthquakes have occurred around the shear zone (see Fig. 1), and the strike directions of the fault planes are oblique to the active fault as is also shown in Fig. 6. This demonstrates the resulting shear stress reduction at the shear zone. High seismic activity was also observed around an active fault called the Hinagu fault, which is located north of the zone. The high seismic activity might be attributed to the strike direction of the Hinagu fault, which is optimum under the obtained stress field and relaxed by the right-lateral motion of the shear zone.
On the basis of the above discussions, we now consider an interpretation for the spatial variation of the stress field. Figure 7 shows a qualitative model of the stress field at Kyushu. According to studies of stress fields in southwestern Honshu (e.g., Matsumoto et al. 2015), the relation among principal stresses is either $\sigma_{\mathrm{Hmin}} \simeq \sigma_{\mathrm{v}}<\sigma_{\mathrm{Hmax}}$ or $\sigma_{\mathrm{Hmin}}<\sigma_{\mathrm{v}}<\sigma_{\mathrm{H} \max }$. The extensional stress might be loaded on Kyushu Island because of the PHS roll-back and/or the extension of the Okinawa trough. Then, $\sigma_{\mathrm{Hmin}}$ decreases, and the extensional stress field dominates. At the shear zone that is considered to be the western extension of the MTL in Honshu, the shear stress is reduced by right-lateral motion; thus, $\sigma_{\mathrm{Hmin}}$ is oriented normal to the shear zone. The $\sigma_{\mathrm{Hmax}}$ might be low in the B- $\mathrm{U}$ area because of the mechanical weak zone around the Aso and Kuju volcanoes, which changes the stress regime to the normal-fault type.

\section{Conclusions}

We have estimated the relative deviatoric stress field on Kyushu Island from the focal mechanisms of shallow earthquakes. The results show that

(1) predominantly, $\sigma_{\mathrm{Hmin}}$ is a major feature of the stress field in Kyushu.

(2) the stress condition is a normal fault, or $\sigma_{\mathrm{Hmax}}$ is equal to $\sigma_{\mathrm{v}}$, in the $\mathrm{B}-\mathrm{U}$ area, which facilitated the graben formation.

(3) the $\sigma_{\mathrm{Hmin}}$ direction is rotated normal to the MTL shear zone, thus suggesting that shear stress reduction occurs as implied by the strain-rate field.

Further studies will take a quantitative approach to model the tectonic stress and strain-rate field.

\section{Competing interests}

The authors declare that they have no competing interests.

\section{Authors' contributions}

SM analyzed the data and designed the research. SN contributed to the discussion of the relation between the stress and strain fields. TO, MM, HS, $\mathrm{HI}, \mathrm{MN}, \mathrm{SY}$, and $\mathrm{YY}$ acquired the data. All the authors have read and approved the final manuscript.

\section{Acknowledgements}

We would like to thank Prof. Takenaka and two anonymous reviewers for their valuable comments that helped us to revise our manuscript. We thank all the staff and students of Kyushu and Kyoto Universities for their effort to obtain good-quality seismic data. We used seismic data from Kyushu, Kyoto, Kagoshima, and Tokyo Universities, the JMA, and Hi-net (NIED). This study was supported by the Observation and Research Program for Prediction of Earthquakes and Volcanic Eruptions (MEXT) and MEXT KAKENHI (No. 26109004)

\section{Author details}

${ }^{1}$ Institute of Seismology and Volcanology, Faculty of Sciences, Kyushu University, Shimabara, Japan. ${ }^{2}$ Graduate School of Science and Technology, Kagoshima University, Kagoshima, Japan. ${ }^{3}$ Aso Volcanological Laboratory, Kyoto University, Kumamoto, Japan. ${ }^{4}$ Earthquake Research Institute, University of Tokyo, Tokyo, Japan. 
Received: 27 March 2015 Accepted: 14 October 2015

Published online: 23 October 2015

\section{References}

Angelier J (1979) Determination of the mean principal directions of stresses for a given fault population. Tectonophysics 56:T17-26. doi:10.1016/00401951(79)90081-7

Angelier J (1984) Tectonic analysis of fault slip data sets. J Geophys Res 89:5835-48

Hardebeck JL, Hauksson E (2001) Crustal stress field in southern California and its implications for fault mechanics. J Giophys Res 106(B10):21859-82

Hardebeck JL, Michael AJ (2004) Stress orientations at intermediate angles to the San Andreas Fault, California. J Geophys Res 109, B11303. doi:10.1029/ 2004JB003239

Hardebeck JL, Michael AJ (2006) Damped regional-scale stress inversions: methodology and examples for southern California and the Coalinga aftershock sequence. J Geophys Res 111, B11310. doi:10.1029/2005JB004144

Hardebeck JL, Shearer PM (2002) A new method for determining first-motion focal mechanisms. Bull Seismo Soc Am 92(6):2264-76

Hori $\mathrm{M}$ et al (2006) Three-dimensional seismic velocity structure as determined by double-difference tomography in and around the focal area of the 2005 west off Fukuoka Prefecture earthquake. Earth Planets Space 58:1621-6

Itoh Y, Kusumoto S, Takemura K. 2014. Evolutionary process of Beppu Bay in central Kyushu, Japan: a quantitative study of the basin-forming process controlled by plate convergence modes. Earth Planets Space. 66:74. doi:10.1186/1880-5981-66-74.

Kamata H, Kodama K (1994) Tectonics of an arc-arc junction: an example from Kyushu Island at the junction of the Southwest Japan Arc and the Ryukyu Arc. Tectonophysics 233:69-81

Kamata H, Kodama K (1999) Volcanic history and tectonics of the Southwest Japan Arc. The Island Arc 8:393-403

Kimura T, Hayami I, Yoshida S (1993) Geology of Japan. University of Tokyo Press, Tokyo, Japan; p 287

Matsumoto S, Uehira K, Matsushima T, Shimizu H (2012) Modeling heterogeneous deviatoric stress field around the hypocentral area of the 2005 Fukuoka earthquake (M7.0) by spatially distributed moment tensors. J Geophys Res 117:B03303. doi:10.1029/2011JB008687

Matsumoto S, Katao H, lio Y (2015) Determining change in the state of stress associated with an earthquake via combined focal mechanism and moment tensor analysis: application to the 2013 Awaji Island earthquake Japan. Tectonophysics 649:58-67

Michael A (1984) Determination of stress from slip data: faults and folds. J Geophys Res 89(B13):11517-26

Nakao S, Miura S, Sato T. 2005. On strain rate deduced from GEONET in Kyushu, Japan, Abstracts 2005 Japan Earth and Planet. Sci. Joint Meeting, D007-013.

National Institute of Advanced Industrial Science and Technology (AIST) (2013) Active Fault Database of Japan, August 22, 2013 version. https:/gbank.gsj.jp/ activefault/index_e_gmap.html.

Nishimura S, Hashimoto M (2006) A model with rigid rotations and slip deficits for the GPS-derived velocity field in southwest Japan. Tectonophysics 421:187-207. doi:10.1016/j.tecto.2006.04.017

Okada A (1980) Quaternary faulting along the Median tectonic line of southwest Japan. Mem Geol Soc Jpn 18:79-108

Saiga A, Matsumoto S, Uehira K, Matsushima T, Shimizu H (2010) Velocity structure in the crust beneath the Kyushu area. Earth Planets Space 62:449-62. doi:10.6047/eps.2010.02.003

Seno T (1999) Syntheses of the regional stress field of the Japanese islands. Island Arc 8:66-79

Shimizu H, Takahashi H, Okada T, Kanazawa T, lio Y, Miyamachi H, Matsushima T, Ichiyanagi M, Uchida N, Iwasaki T, Katao H, Goto K, Matsumoto S, Hirata N, Nakao S, Uehira K, Shinohara M, Yakiwara H, Kame N, Urabe T, Matsuwo N, Yamada T, Watanabe A, Nakahigashi K, Enescu B, Uchida K, Hashimoto S, Hirano S, Yagi T, Kohno Y, Ueno T, Saito M, Hori M (2006) Aftershock seismicity and fault structure of the 2005 West Off Fukuoka Prefecture Earthquake (MJMA7.0) derived from urgent joint observations. Earth Planets Space 58:1599-604

Terakawa T, Matsu'ura M (2010) 3-D tectonic stress fields in and around Japan inverted from CMT data of seismic events. Tectonics 29, TC6008. doi:10.1029/2009TC002626

Townend J, Zoback MD (2001) Implications of earthquake focal mechanisms for the frictional strength of the San Andreas fault system. In: Holdsworth RE,
Strachan RA, Macloughlin J, Knipe RJ, (Eds.). The Nature and Significance of Fault Zone Weakening, vol. 186. Geological Society of London Special Publication; pp.13-21.

Townend J, Zoback MD (2004) Regional tectonic stress near the San Andreas Fault in central and southern California. Geophys Res Lett 31:L15S11. doi:10.1029/2003GL018918

Townend J, Zoback MD (2006) Stress, strain, and mountain building in central Japan. J Geophys Res 111, B03411. doi:10.1029/2005JB003759

Wallace LM, Ellis S, Miyao K, Miura S, Beavan J, Goto J (2009) Enigmatic, highly active left-lateral shear zone in southwest Japan explained by aseismic ridge collision. Geology 37(2):143-6. doi:10.1130/G25221A.1

Yamaji A (2003) Slab rollback suggested by latest Miocene to Pliocene forarc stress and migration of volcanic front in southern Kyushu, northern Ryukyu Arc. Tectonophysics 346:9-24. doi:10.1016/S0040-1951(03)00047-7

Zoback MD et al (1987) New evidence for the state of stress on the San Andreas fault system. Science 238:1105-11

\section{Submit your manuscript to a SpringerOpen ${ }^{\odot}$ journal and benefit from:}

- Convenient online submission

Rigorous peer review

- Immediate publication on acceptance

- Open access: articles freely available online

- High visibility within the field

- Retaining the copyright to your article

Submit your next manuscript at $>$ springeropen.com 